Pacific

Journal of

Mathematics

\title{
CAPILLARY SURFACES IN WEDGE DOMAINS
}

Jin TZu Chen, Robert Finn ANd ERICH Miersemann 


\title{
CAPILLARY SURFACES IN WEDGE DOMAINS
}

\author{
Jin Tzu Chen, Robert Finn And ERich Miersemann
}

\begin{abstract}
We consider capillary surfaces $u(x, y)$ in a vertical tube whose sections contain a corner (wedge) with opening $2 \alpha, 0<2 \alpha<2 \pi$, and with contact angle data $\gamma_{1}, \gamma_{2}$ on the adjacent sides. We interpret geometrically and improve conditions of Lancaster and Siegel on criteria for boundedness of solutions. In the case that the solutions are unbounded we give growth estimates and in the classical case of downward gravity field a power representation asymptotic to any prescribed order. The coefficients of this expansion are independent of the particular solution considered.
\end{abstract}

\section{Introduction}

We are interested in the behavior of the upper surface of liquid in a vertical capillary tube whose horizontal sections contain a sharp corner, so that the tube appears near a vertical line as a wedge; see photo on the right, taken from Miersemann [2008], for a physical example. Observations of the behavior of fluid in a vertical wedge with small opening angle can be traced at least to Brook Taylor [1712].

We restrict our attention to surfaces $\mathcal{S}$ defined as graphs $u(x, y)$ over a base domain $\Omega \subset \mathbb{R}^{2}$ containing a corner made by rectilinear segments $\Sigma_{1}, \Sigma_{2}$ meeting at an angle $2 \alpha$, with $0<\alpha<\pi$; see Figure 1 on the next page. We may assume that $\Sigma_{1}$ intersects $\Sigma_{2}$ in the origin $O$ of coordinates, and that the positive $x$-axis

bisects the angle between them. The vertical planes containing $\Sigma_{1}, \Sigma_{2}$ may be of different but respectively homogeneous materials, so that the contact angles $\gamma_{1}, \gamma_{2}$

MSC2000: primary 76B45; secondary 53A10, 49Q10.

Keywords: capillarity, mean curvature, corner behavior, comparison method. 


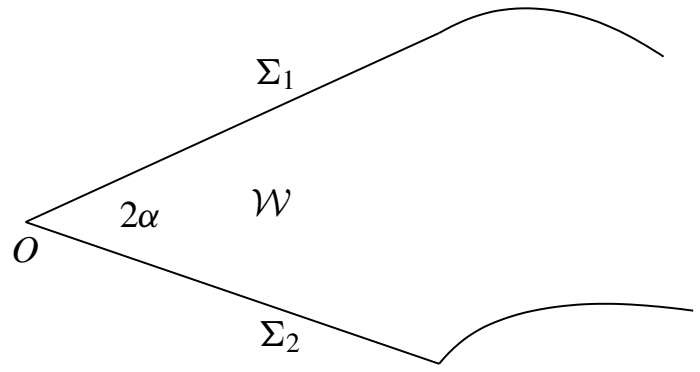

Figure 1. Cross section of the tube.

in which $\mathcal{S}$ meets the planes are (perhaps different) constants on each plane. Set

$$
T u \equiv D u / \sqrt{1+|D u|^{2}} .
$$

We are led to the boundary data problem

$$
\begin{aligned}
\operatorname{div}(T u)(x, y) & =f(x, y, u(x, y)) \text { for }(x, y) \in \Omega, \\
v \cdot T u & =\cos \gamma_{1} \text { on } \Sigma_{1}, \\
v \cdot T u & =\cos \gamma_{2} \text { on } \Sigma_{2} .
\end{aligned}
$$

Here $f(x, y, s)$ is a prescribed function and $v$ is unit exterior normal. The system (1-1) arose from studies of Young [1805], of Laplace [1805, 1806], and of Gauss [1830], who considered the "classical case" $f(x, y, s) \equiv \kappa s+2 H$, with $\kappa \geq 0$ a physical constant, and $2 H$ a Lagrange parameter arising from an eventual volume constraint. The case $\kappa>0$ corresponds to a vertically downward uniform gravity field, while $\kappa=0$ means absence of gravity. We note that the divergence expression in (1-1) is exactly twice the mean curvature of $\mathcal{S}$, so the problem can be viewed as the purely geometrical one of determining a surface of prescribed mean curvature that meets prescribed bounding walls in a prescribed angle.

It is known that very varied kinds of behavior can occur in the classical case, depending on the particular geometry and on the data. Concus and Finn [1969, 1974] showed in the case $\gamma_{1}=\gamma_{2}=\gamma$ that a discontinuous transition in behavior can occur, as $\gamma$ crosses the critical value $\frac{\pi}{2}-\alpha$. They showed also that if $\kappa>0$ and $2 \alpha>\pi$ then all solutions are bounded at $O$. Korevaar [1980] showed that if $\kappa>0$ and $2 \alpha>\pi$ then the (bounded) solution can be discontinuous at $O$. For general $\gamma_{1}, \gamma_{2}$ Concus and Finn [1996] introduced the rectangle $\mathcal{R}$ and domains $\mathcal{D}_{1}, \mathcal{D}_{2}$ (see Figure 2) and showed that if $\kappa=0$ then solutions fail to exist if the data lie in the $\mathcal{D}_{1}$ domains, and must have discontinuous unit normals at $O$ if the data are in the $\mathcal{D}_{2}$ domains. Finn [1996] showed that solutions can exist for any data in $\mathcal{D}_{2}$. Lancaster and Siegel [1997] showed that solutions exhibit "fans" of directions of approach to $O$ in which the radial limits are constant, and showed 


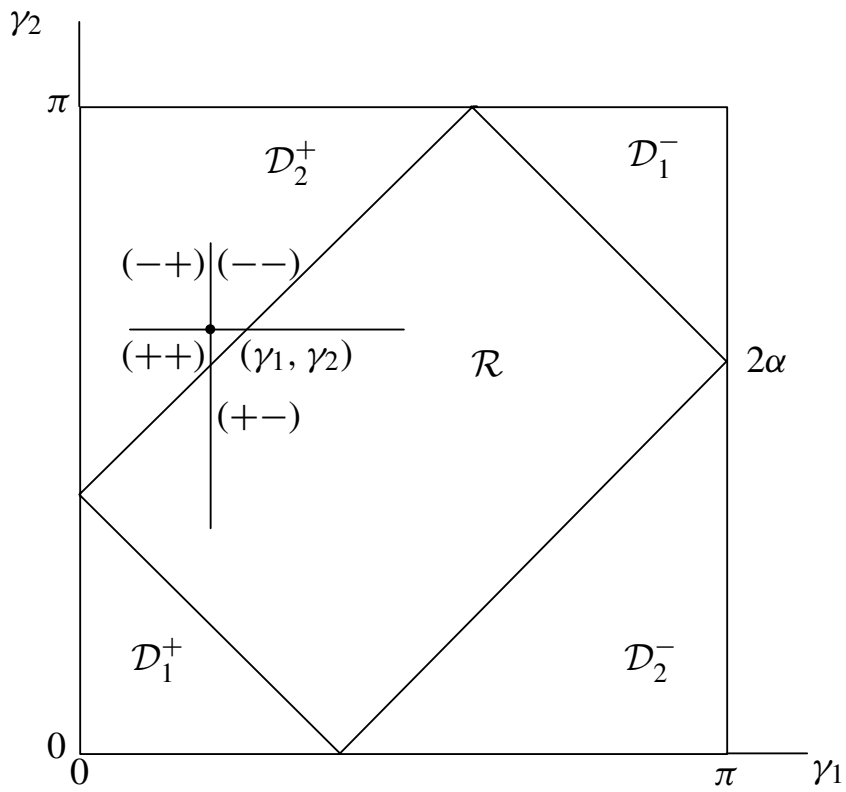

Figure 2. Data rectangle $\mathcal{R}$ of regularity; notational designations.

that if $2 \alpha<\pi$ and if the data are in $\mathcal{R}$ then there is only a single fan, which covers all directions. As a consequence, they showed continuity at $O$ of all solutions in that case. Concus and Finn [1996] indicated independently another approach to that result.

Simon [1980] showed that if $\gamma_{1}=\gamma_{2}=\gamma$, if $\alpha<\pi / 2$ and if $\alpha+\gamma>\pi / 2$ then every solution is differentiable up to $O$. Tam [1986] extended that result to $\alpha+\gamma \geq \pi / 2$. Lieberman [1988] and Miersemann [1989] showed that such solutions are Hölder differentiable at $O$. Huff and McCuan [2006] gave examples to show that solutions need not be twice differentiable at $O$.

In a gravitational field $\kappa>0$ with $\gamma_{1}=\gamma_{2}=\gamma$, Concus and Finn [1969, 1974] showed that for data in $\mathcal{D}_{1}$ all solutions become unbounded at $O$ like $1 / r$, where $r=\sqrt{x^{2}+y^{2}}$. This result was extended by Miersemann [1993] to an expansion in powers of $r$ that is asymptotic to any prescribed finite order, and whose coefficients and domain of validity are independent of the particular solution considered. In the present work we show that a result of the same character applies to all solutions arising from data in $\mathcal{D}_{1}$ domains.

According to a conjecture of Concus and Finn, every solution is discontinuous at $O$ if the data are in $\mathcal{D}_{2}$ domains. A proof of the conjecture was announced by Lancaster [ $\geq 2008]$.

In the present work, we investigate the consequences that varying kinds of behavior of $f(x, y, s)$ have on the behavior of solutions of (1-1) that can arise. 
Notably, we establish boundedness of solutions in some cases and estimate growth rates at the vertex of the wedge when unbounded solutions appear. We study both protruding and reentrant corners, which lead to strikingly different behavior patterns.

\section{Boundedness of solutions; protruding corners}

In this section we give a new formulation and proof of [Lancaster and Siegel 1997, Proposition 1] concerning the boundedness of solutions at corner points of the boundary. Our approach emphasizes the essential geometric content of the result, and provides notable simplification in detail, with some explicit estimates. Additionally, we extend the result to a general class of equations (1-1) and include configurations for which the solutions can be unbounded, showing that they are subject to severe restraints in the rate of growth. Our hypotheses on boundary angles $\gamma_{1}, \gamma_{2}$ and also on boundary configuration are in one sense more restrictive than those introduced in [Lancaster and Siegel 1997], permitting us to dispose of a restriction on the angles that could distract attention from the underlying geometrical relations. Specifically we restrict attention to corners determined by locally rectilinear boundaries $\Sigma$ and to data $\gamma_{1}, \gamma_{2}$ that are locally constant near the corner point. These restrictions permit in another sense more generality, by allowing us to state our results for the entire closed ranges of the regions considered for the boundary data. The modifications that are needed to include all configurations addressed in [Lancaster and Siegel 1997] are indicated by the discussion in [Finn 1996]. We thus consider solutions of (1-1) defined locally in a wedge region $\mathcal{W}$, in a neighborhood $\mathcal{N} \subset \mathcal{W}$ of the vertex $O$. For conceptual clarity we assume that all derivatives entering in the expressions encountered exist and are continuous, both within the (open) $\mathcal{N}$ and on the bounding walls $\Sigma_{j}$, with $O$ delated. This regularity is implied under much weaker conditions, that is, the "variational solutions" introduced in [Finn 1986, Chapter 7].

We distinguish between protruding corners, for which the opening angle $2 \alpha<\pi$, and reentrant corners, for which $2 \alpha>\pi$. Concus and Finn [1996] showed for a protruding corner that a surface $u(x, y)$ defined in some $\mathcal{N}$ with continuous normal vector up to $O$ can exist only if the data $\left(\gamma_{1}, \gamma_{2}\right)$ at $O$ lie in the closed rectangle $\mathcal{R}$ inscribed in the square $\mathcal{Q}$ of Figure 2 .

Such surfaces do exist throughout the open rectangle, and on the side $\mathcal{L}_{1}^{+}$joining $\mathcal{R}$ to $\mathcal{D}_{1}^{+}$, but not on the other sides of $\mathcal{R}$. These are general properties of surfaces and have nothing to do with the equation. The analogous rectangle for a reentrant corner is characterized in [Shi 2006]. These observations permit us to construct surfaces of constant mean curvature, as lower or upper hemispheres or as planes, for any prescribed data in $\mathcal{R}$. We will use these surfaces as comparison surfaces to 
bound the heights of general solutions of (1-1) achieving prescribed data near the vertex of a wedge.

Corresponding to any point $\left(\gamma_{1}, \gamma_{2}\right) \in \mathcal{Q}$ we introduce the four regions $(--)$, $(-+),(+-),(++)$ in $\mathcal{Q}$ determined by the coordinate lines through that point; see Figure 2. We introduce four representative hypotheses on $f$ :

Hypothesis $\mathbf{A}^{+}$. Given $M>0$, there exists $U(M)$ such that $f(x, y, s)>M$ whenever $s>U(M)$.

Hypothesis $\mathbf{A}^{-}$. Given $M>0$, there exists $U(M)$ such that $f(x, y, s)<-M$ whenever $s<-U(M)$.

Hypothesis $\mathbf{B}^{+} .0<m<f(x, y, s)<M<\infty$ for all $(x, y, s)$.

Hypothesis $\mathbf{B}^{-} .0>-m>f(x, y, s)>-M>-\infty$ for all $(x, y, s)$.

If $f$ is strictly increasing in $s$, we write $f \in \mathcal{M}$. We consider separately the cases of protruding and of reentrant corners. We normalize all contact angles $\gamma$ that occur to the range $0<\gamma<\pi$. We say that data $\gamma^{(a)}$ majorize data $\gamma^{(b)}$ if $\gamma^{(a)} \leq \gamma^{(b)}$ and that $\gamma^{(a)}$ minorize $\gamma^{(b)}$ if $\gamma^{(a)} \geq \gamma^{(b)}$. For physical problems, one expects that if $\gamma^{(a)}$ majorize $\gamma^{(b)}$ at all boundary points of a domain $\Omega$ of definition then the corresponding solutions satisfy $u_{a} \geq u_{b}$ throughout $\Omega$. However, that may not be the case under the present hypotheses, as monotonicity of $f$ in $s$ is not assumed. That would also clearly not be the case when $f$ is independent of $s$.

We base the present study on the observation that for any data pair $\left(\gamma_{1}, \gamma_{2}\right)$ on the respective wedge sides, which lies in $\mathcal{R} \cup \mathcal{D}_{2}^{ \pm} \cup \mathcal{L}_{2}^{ \pm}$, a pair $\left(\gamma_{1}^{+}, \gamma_{2}^{+}\right) \in \mathcal{R}$ can be found that majorizes both $\gamma_{1}$ and $\gamma_{2}$, and another pair $\left(\gamma_{1}^{-}, \gamma_{2}^{-}\right) \in \mathcal{R}$ can be found that minorizes both $\gamma_{1}$ and $\gamma_{2}$. One sees that immediately from Figure 2; the minorizing point appears in the $(++)$ domain, the majorizing point in the $(--)$ domain. This will permit us to find both upper and lower bounds for solutions arising from such data. That is however not the case for other data. Data in $\mathcal{D}_{1}^{+} \cup \mathcal{L}_{1}^{+}$ can only be minorized by data in $\mathcal{R}$, while data in $\mathcal{D}_{1}^{-} \cup \mathcal{L}_{1}^{-}$can only be majorized within $\mathcal{R}$. That circumstance leads to essential differences in the kinds of bounds that can be established for these domains.

We will obtain bounds by way of comparison theorems, in terms of comparison surfaces $v(x, y)$ with data that majorize (or minorize) on both segments. We will also need classical comparison methods deriving from numerical values of the solutions on particular segments. The methods and results vary according to which of the five indicated subregions of $\mathcal{Q}$ in Figure 2 contains the data pair $\left(\gamma_{1}, \gamma_{2}\right)$ for the given solution on the two segments abutting on $O$. We consider separately the five cases, relative to the four indicated hypotheses. Our central weapons will be variants of the general comparison principle [Finn 1986, Theorem 5.1], designed to accommodate situations in which $f$ is not monotone in $s$. 


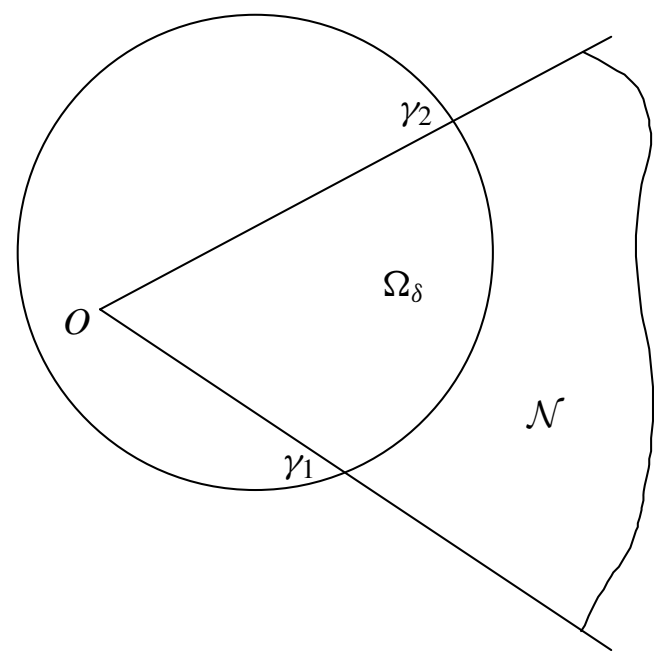

Figure 3. Domain for comparison hemispheres; $\left(\gamma_{1}, \gamma_{2}\right)$.

\section{A. Data $\left(\gamma_{1}, \gamma_{2}\right) \in \mathcal{R}$.}

a. Hypothesis $\mathbf{A}^{+}$. We choose as comparison surface a lower hemisphere $v(x, y)$ of radius $\delta$ small enough that it can be positioned to meet the two boundary segments in the precise angles $\left(\gamma_{1}, \gamma_{2}\right)$, and such that the subdomain $\Omega_{\delta}$ cut off by its equatorial circle $\Sigma_{\delta}$ determines a neighborhood $\mathcal{N}_{\delta} \subset \mathcal{N}$ of $O$; see Figure 3 . It is shown in Concus and Finn [1996] that this construction is possible for all $\left(\gamma_{1}, \gamma_{2}\right) \in \mathcal{R}$ and for no data exterior to $\mathcal{R}$. We then have

$$
\operatorname{div}(T v)(x, y)=\frac{2}{\delta}
$$

in $\Omega_{\delta} \cap \mathcal{N}$, with $v \cdot T v=v \cdot T u$ on the two wedge boundary segments.

Setting

$$
F(x, y, u(x, y))=\min _{s \geq u(x, y)} f(x, y, s)
$$

we find

$$
\operatorname{div}(T u)(x, y) \geq F(x, y, u(x, y))
$$

with $F(x, y, s)$ satisfying Hypothesis $\mathrm{A}^{+}$, and nondecreasing in $s$. Integration by parts yields

$$
\begin{gathered}
\int_{\Omega_{\delta} \cap \mathcal{N}}(D \eta \cdot(T v-T u)+\eta(F(x, y, v)-F(x, y, u))) d \Omega \\
\geq \oint_{\Sigma_{\delta} \cap \mathcal{N}} \eta(v \cdot T v-v \cdot T u) d s+\int_{\Omega_{\delta} \cap \mathcal{N}} \eta(F(x, y, v)-2 / \delta) d \Omega
\end{gathered}
$$

for all $\eta \in \mathcal{Q}\left(\Omega_{\delta}\right) \equiv L^{\infty} \cap H^{1,1}\left(\Omega_{\delta}\right)$. 
Since $v$ is vertical on $\Sigma_{\delta}$, we find $v \cdot T v=1>v \cdot T u$ on $\Sigma_{\delta}$. We are free to raise or lower the hemisphere at will. We choose the height of $v(x, y)$ so that at its lowest point in the closure of $\mathcal{N}$, there holds

$$
v=M_{\delta}=\max \{t: F(x, y, t) \leq 2 / \delta\} .
$$

With this normalization, we then find that the two integrals on the right in (2-1) are positive for any positive $\eta$ in the given class; hence this holds also for the first integral in (2-1). From [Athanassenas and Finn 2006, Lemma 2.1] we conclude that $u<v$ throughout $\Omega_{\delta}$. Since the total height change of $v$ in this domain does not exceed $\delta$ we conclude finally

$$
u(x, y)<M_{\delta}+\delta
$$

throughout $\Omega_{\delta}$. This provides an explicit upper bound for $u$ at the corner point, for any data in $\mathcal{R}$.

b. Hypothesis $\mathbf{A}^{-}$. With analogous definitions and procedure as in the preceding case, we obtain, for a suitable $\bar{M}_{\delta}$,

$$
u(x, y)>-\bar{M}_{\delta}-\delta
$$

throughout $\Omega_{\delta}$.

In the case of particular interest $f \in \mathrm{A}^{+} \cup \mathrm{A}^{-} \cup \mathcal{M}$, Lancaster and Siegel [1997] showed that if the data are in $\mathcal{R}$ then $u$ is continuous at $O$. That is the case for example for the classical capillary equation

$$
\operatorname{div}(T u)(x, y)=\kappa u(x, y)+\lambda, \quad \kappa>0 .
$$

c. Hypothesis $\mathbf{B}^{+}$. If $f$ is bounded in $s$, explicit bounds on the solution height cannot be expected from local information. As an example, the choice $f \equiv$ const. leads to solutions that continue to satisfy the equation and boundary conditions on addition of arbitrary constants. We may however assume a solution given in $\mathcal{N}$, and infer bounds up to the vertex, in terms of bounds for the solution at points removed from the vertex. Since the data are in $\mathcal{R}$, we may position a lower hemisphere $\mathcal{H}: v(x, y)$ so as to achieve that data locally on the two wedge segments; we choose the radius of $\mathcal{H}$ to be $R>1 / m$, thus achieving a configuration as in Figure 4. We construct a segment $L$ as in that figure. By hypothesis, $u$ is bounded uniformly on compact subsets of $L$; we show initially that $u$ is bounded up to the contact points with the wedge. To do that, we introduce another lower hemisphere $\mathcal{H}^{*}: v^{*}(x, y)$ of the same radius, as indicated in Figure 5, of which a segment $\mathcal{D}$ of the equatorial disk is cut off as indicated, so as to lie entirely in $\mathcal{N}$. We raise $v^{*}$ so that $v^{*}>u$ on $L^{*}$, and we assert that then $v^{*}>u$ throughout $\mathcal{D}$. In fact, were a subdomain 


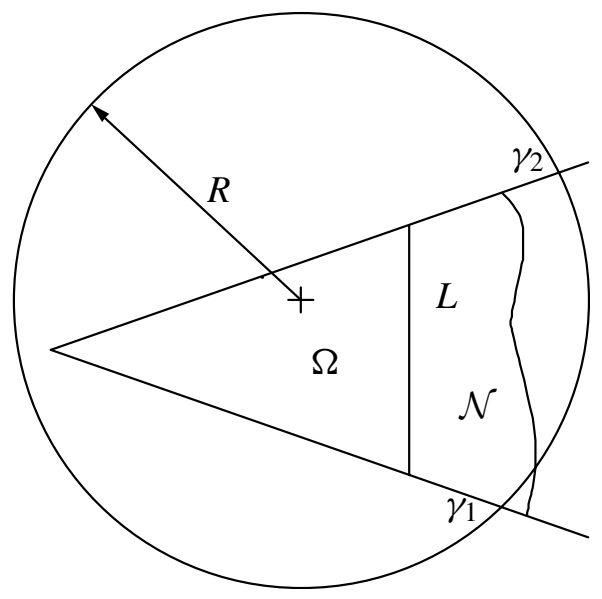

Figure 4. Configuration for data in $\mathcal{R}$, Hypothesis $\mathrm{B}^{+}$.

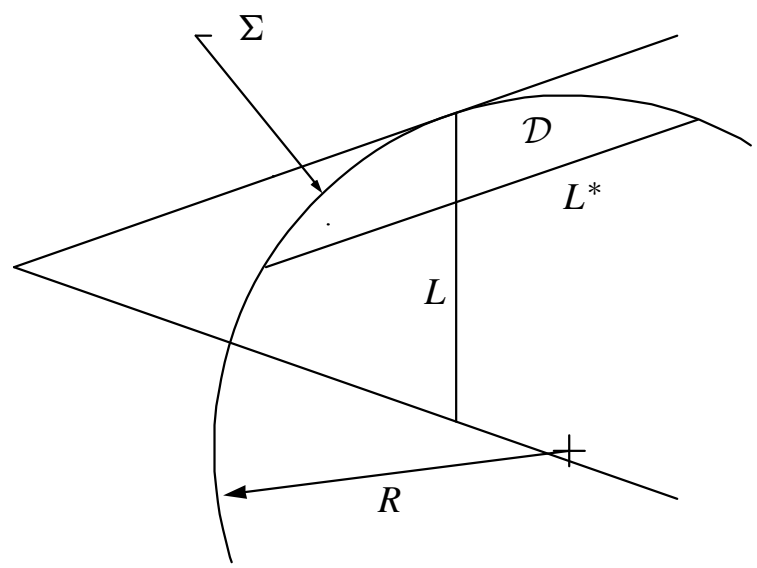

Figure 5. Bound on $L$ near wedge boundary, in terms of interior bounds.

$\Omega \subset \mathcal{D}$ to appear, in which $u>v^{*}$, we would find, for any $A>0$,

$$
\begin{gathered}
\int_{\Omega} \eta(f(x, y, u)-m) d \Omega \\
=\oint_{\Sigma} \eta(v \cdot T u-v \cdot T v) d s-\int_{\Omega} \nabla \eta \cdot\left(T u-T v^{*}\right) d \Omega,
\end{gathered}
$$

with

$$
\eta=\left\{\begin{aligned}
0 & : \quad u-v^{*} \leq 0, \\
u-v^{*} & : 0 \leq u-v^{*} \leq A, \\
A & : \quad u-v^{*}>A .
\end{aligned}\right.
$$


Hypothesis $\mathrm{B}^{+}$implies that the left side of (2-4) is positive. Since $v \cdot T v=1$ on $\Sigma$ while $v \cdot T u<1$ at points distinct from $O$, the first integral on the right is negative. As is known (see the proof of [Finn 1986, Theorem 5.1]) the integrand in the final term is nonnegative and vanishes at points for which $0<\eta<A$ only if $\nabla u=\nabla v^{*}$. This contradiction shows that $u<v$ in the segment; the same reasoning applies also at the other end of $L$, and we conclude that $u$ is bounded above on $L$.

Returning to Figure 4, we may now raise the hemisphere $\mathcal{H}$ until $v>u$ on $L$. Since the boundary conditions for $v$ are identical on the wedge segments to those for $u$, a reasoning as above yields again $u<v$ in the region $\Omega$. We thus obtain the existence of an upper bound for $u$, although the procedure does not yield an explicit bound.

d. Hypothesis $\mathbf{B}^{-}$. A reasoning symmetric to the one above yields that $u$ is bounded below.

Remark 1. The results above are not vacuous, in the sense that solutions satisfying the hypotheses do in fact exist in particular cases. The explicit comparison surfaces chosen provide particular solutions when $f$ is constant; existence for particular cases $\mathrm{A}^{+}$and $\mathrm{A}^{-}$is demonstrated in Emmer [1973].

Remark 2. Variable data on the wedge segments are admissible, when they can be majorized by admissible constant data in the case of upper bounds, or minorized in the case of lower bounds.

Remark 3. The requirements in the Hypotheses $\mathrm{B}^{+}$and $\mathrm{B}^{-}$that $f$ be bounded from zero are essential. One can see that from the example $u=1 /(x+y)$, which satisfies an equation of the form (1-1) with

$$
f(x, y, s)=\frac{4 s^{3}}{\left(1+2 s^{4}\right)^{3 / 2}}
$$

in the wedge domain $x>0, y>0$, for which $\alpha=\pi / 4$. The surface intersects the bounding walls in the angles

$$
\gamma_{1}(u)=\gamma_{2}(u)=\gamma(u), \quad \text { with } \cos \gamma=\frac{u^{2}}{\sqrt{1+2 u^{4}}}<\frac{1}{\sqrt{2}} .
$$

The corresponding angles are thus seen to lie in $\mathcal{R}$, and are all majorized by the limiting position $\gamma_{1}=\gamma_{2}=\pi / 4$ on the boundary of $\mathcal{D}_{1}^{-}$. For this solution, $f$ is positive but tends to zero with increasing $s$. Thus all requirements for Hypothesis $\mathrm{B}^{+}$ are satisfied except for the bound below of $f$ from zero, and the solution is clearly unbounded. From a geometrical point of view, our hypotheses exclude the kinds of behavior exemplified by minimal surfaces, which can in some ways be qualitatively very different from the examples considered here. 


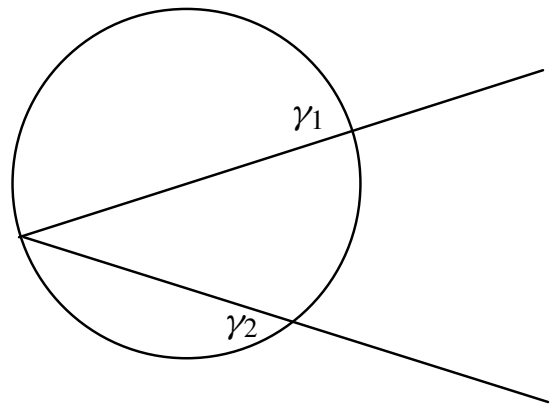

Figure 6. Data on $\mathcal{L}_{1}^{+}$.

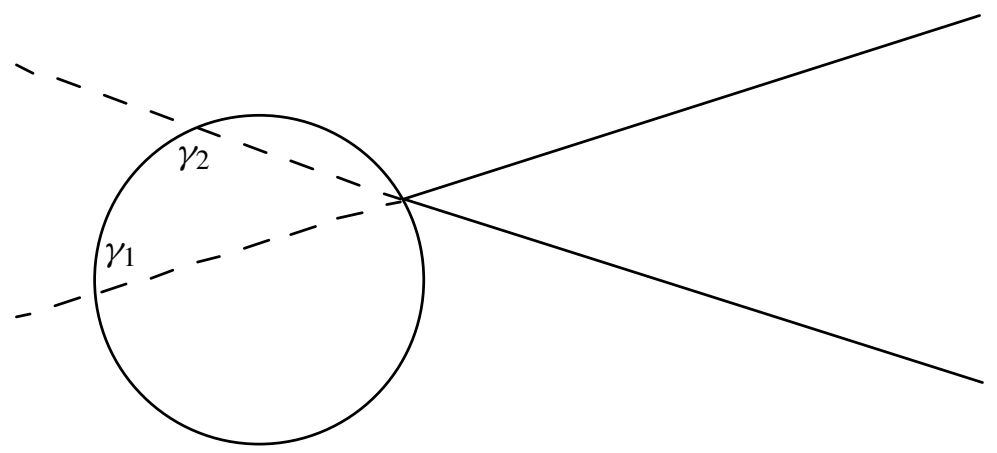

Figure 7. Data on $\mathcal{L}_{1}^{-}$.

We are now prepared to dispose of all other cases.

2B. Data $\left(\gamma_{1}, \gamma_{2}\right) \in \mathcal{L}_{1}^{ \pm}$.

a. Hypothesis $\mathbf{A}^{+}$, data on $\mathcal{L}_{1}^{+}$. The equatorial circle for a lower comparison hemisphere takes the form indicated in Figure 6, with the prescribed data assumed exactly. No other such comparison is possible; however the result (2-2) continues to hold using that data. In Section 3 we shall see that a discontinuous change in behavior occurs when the data move even infinitesimally into $\mathcal{D}_{1}^{+}$.

b. Hypothesis $\mathbf{A}^{+}$, data on $\mathcal{L}_{1}^{-}$. In this case the equatorial circle is positioned as in Figure 7, and covers no neighborhood of $O$ in the wedge. Following the reasoning of Section 3 for this limiting configuration, we see that an analogue of Theorem 2.1 of Section $2 \mathrm{C}$ governs the behavior, and that a bound of the form (2-3) applies. The case of data on $\mathcal{L}_{2}^{ \pm}$, is covered in Section $2 \mathrm{C}$.

2C. Data $\left(\boldsymbol{\gamma}_{1}, \boldsymbol{\gamma}_{2}\right) \in \mathcal{D}_{2}^{ \pm} \cup \mathcal{L}_{2}^{ \pm}$. Remarkable local properties of the solutions in such configurations are characterized in Lancaster and Siegel [1997, 1996]. With regard simply to bounds of the solutions, this case is essentially subsumed under Section $2 \mathrm{~A}$. The remarks preceding that section point out that for any point $\left(\gamma_{1}, \gamma_{2}\right)$ 


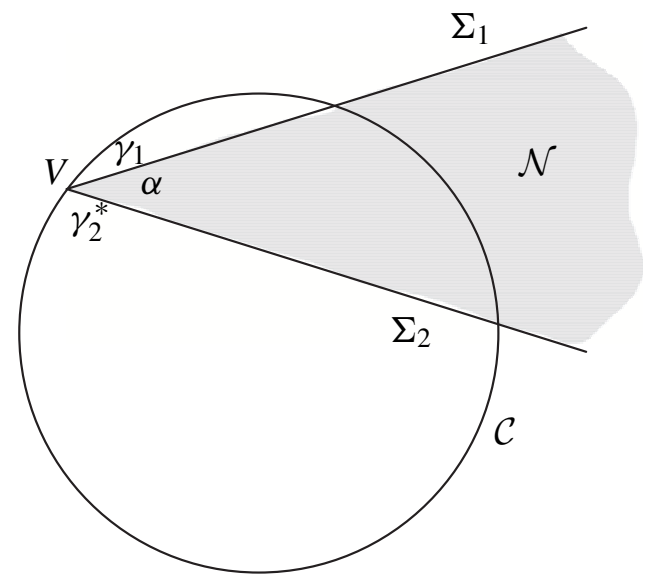

Figure 8. Construction of particular majorizing data when $\left(\gamma_{1}, \gamma_{2}\right) \in \mathcal{D}_{2}^{ \pm}$.

in these sets, both majorizing and minorizing data in $\mathcal{R}$ can be found, thus ensuring boundedness of solutions under our hypotheses. All relations used in Section 2A strengthen under use of such comparison data. As an explicit way to choose comparison data we may proceed as follows: In $\mathcal{D}_{2}^{+} \cup L_{2}^{+}$we have $\gamma_{2}-\gamma_{1} \geq \pi-2 \alpha$. Consider a circle $\mathcal{C}$ passing through the vertex of the wedge, meeting the side $\Sigma_{1}$ in angle $\gamma_{1}$, and of small enough radius that the portion of $\mathcal{C}$ inside the wedge lies in $\mathcal{N}$; see Figure 8 . For the angle $\gamma_{2}^{*}$ with which $\mathcal{C}$ meets $\Sigma_{2}$, we find

$$
\gamma_{2}^{*}=\pi-2 \alpha-\gamma_{1}<\pi-2 \alpha+\gamma_{1} \leq \gamma_{2} .
$$

Thus, the pair $\left(\gamma_{1}, \gamma_{2}^{*}\right)$ majorizes the given angles $\left(\gamma_{1}, \gamma_{2}\right)$, and we see that a lower hemisphere with $\mathcal{C}$ as equatorial circle provides a majorizing solution in the corner. By [Concus and Finn 1996, Theorem 1] the existence of such a $\mathcal{C}$ is equivalent to $\left(\gamma_{1}, \gamma_{2}^{*}\right) \in \mathcal{R}$. Similarly, for data in $\mathcal{D}_{2}^{-}$the pair $\left(\gamma_{1}^{*}, \gamma_{2}\right)$ majorizes the given angles $\left(\gamma_{1}, \gamma_{2}\right)$.

To find minorizing data, observe that minorizing data $\left(\gamma_{1}, \gamma_{2}\right)$ is equivalent to majorizing data $\left(\tau_{1}, \tau_{2}\right)$ with $\tau_{1}=\pi-\gamma_{1}, \tau_{2}=\pi-\gamma_{2}$. But $\left(\gamma_{1}, \gamma_{2}\right) \in \mathcal{D}_{2}^{+}\left(\mathcal{D}_{2}^{-}\right)$is equivalent to $\left(\tau_{1}, \tau_{2}\right) \in \mathcal{D}_{2}^{-}\left(\mathcal{D}_{2}^{+}\right)$. We conclude:

Theorem 2.1. All bounds asserted in Section $2 A$ for data in $\mathcal{R}$ continue to be valid for any data in $\mathcal{D}_{2}^{ \pm} \cup \mathcal{L}_{2}^{ \pm}$.

For such data, the results hold even in somewhat strengthened form, as one sees by following the details of the demonstrations in that section. Nevertheless, the surfaces can exhibit exotic behavior at the vertex $O$ of the wedge. By [Concus and Finn 1996, Theorem 1], solutions with data in $\mathcal{D}_{2}^{ \pm}$cannot have continuous derivatives up to $O$. 


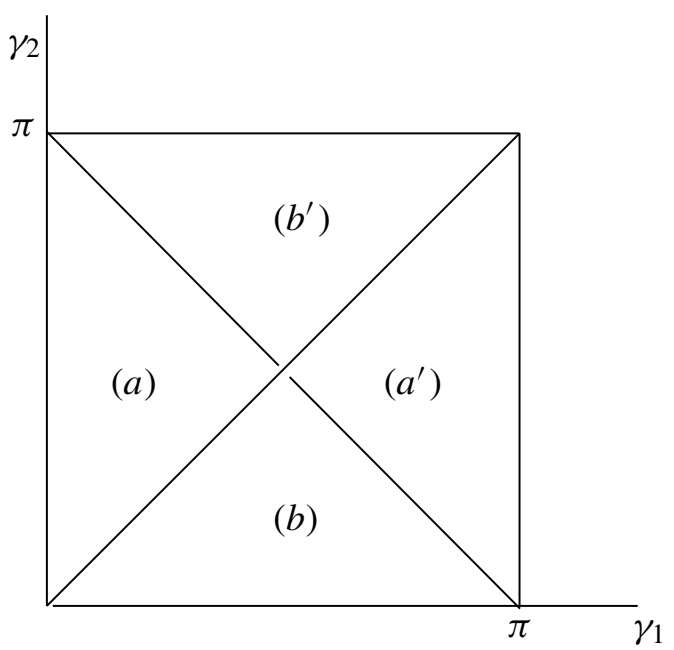

Figure 9. Decomposition of the angle quadrangle $\mathcal{Q}$.

The conjecture of Concus and Finn that every such solution is discontinuous at $O$ formed the basis for a deep investigation by Danzhu Shi [2006], who used it as the foundation for a study of the reentrant corner case; see Section 4.

\section{Growth estimates; protruding corners; data $\left(\gamma_{1}, \gamma_{2}\right) \in \mathcal{D}_{1}^{ \pm}$}

a. Hypothesis $\mathbf{A}^{+}$. If $\left(\gamma_{1}, \gamma_{2}\right) \notin \mathcal{R}$ the procedure we used for data in that domain no longer applies, as the comparison hemisphere no longer covers the vertex $O$, see for example, the discussion in [Finn 1996, Appendix]. The procedure can however be adapted to yield a growth estimate for solutions, when $\left(\gamma_{1}, \gamma_{2}\right)$ lies in a $\mathcal{D}_{1}$ domain.

We begin by introducing some notational simplifications. These preparations are of general interest, for any data. Observe that the quadrangle $\mathcal{Q}$ with the symmetry point $(\pi / 2, \pi / 2)$ delated can be written as the union of four sets (see Figure 9):

(a) $0 \leq \gamma_{1}<\pi / 2, \gamma_{1} \leq \gamma_{2} \leq \pi-\gamma_{1}$,

(b) $0 \leq \gamma_{2}<\pi / 2, \gamma_{2} \leq \gamma_{1} \leq \pi-\gamma_{2}$,

(a') $\left(\gamma_{1}, \gamma_{2}\right)$ of (a) is replaced by $\left(\pi-\gamma_{1}, \pi-\gamma_{2}\right)$,

(b') $\left(\gamma_{1}, \gamma_{2}\right)$ of (b) is replaced by $\left(\pi-\gamma_{1}, \pi-\gamma_{2}\right)$.

The problem (1-1) with data in the upper triangles (a') or (b') changes to a problem with data in the lower triangles (a) or (b) or vice versa for $v:=-u$ if we replace the right hand side $f$ by $-f(x, y,-v)$. Note that in the classical case $f=\kappa u$ this is equal to $\kappa v$. It follows that we may assume without loss of generality 


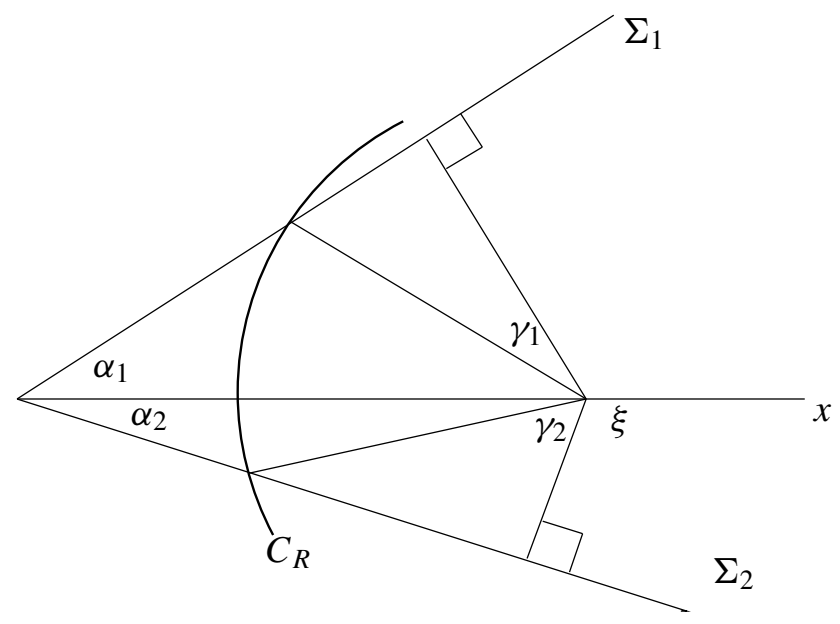

Figure 10. Construction of comparison hemisphere.

that

$$
0 \leq \gamma_{1}<\frac{\pi}{2} \text { and } \gamma_{1} \leq \gamma_{2} \leq \pi-\gamma_{1},
$$

keeping in mind the change of the right hand side of (1-1) if data are in the upper triangle $\gamma_{1}+\gamma_{2}>\pi$. If the data are in (b), then we have to replace $\gamma_{1}$ by $\gamma_{2}$ and vice versa.

We generalize the circle construction of Concus and Finn [1974] to different contact angles as shown in Figure 10. Here $C_{R}$ is a circle of radius $R$ and center at $(\xi, 0)$, where $\xi=k R$. If

$$
k:=\frac{\cos \gamma_{1}}{\sin \alpha_{1}}=\frac{\cos \gamma_{2}}{\sin \alpha_{2}},
$$

where here $\alpha_{1}, \alpha_{2} \neq 0$ and $\alpha_{2}$ is negative if $\gamma_{2}>\pi / 2$. Let $k>1$ and $\alpha_{1}<\pi / 2$, the circle makes the correct angles with $\Sigma_{1}, \Sigma_{2}$, respectively. In particular, the circle $C_{R}$ cuts $\Sigma_{1}$ and $\Sigma_{2}$ and remains bounded away from the vertex $O$. The following lemmata show that such a construction is possible.

Set $\theta:=2 \alpha$ and assume $0<\theta<\pi$ (protruding corner).

Lemma 3.1. Given $0 \leq \gamma_{1}<\pi / 2, \gamma_{1} \leq \gamma_{2} \leq \pi-\gamma_{1}$. Then there is a partition of $\theta$ by $\gamma_{1}, \gamma_{2}$, namely $\theta=\alpha_{1}+\alpha_{2}$, such that

$$
\frac{\cos \gamma_{1}}{\sin \alpha_{1}}=\frac{\cos \gamma_{2}}{\sin \alpha_{2}},
$$

where $\alpha_{1}$ is the unique solution of

$$
x \in(0, \pi): \frac{\sin (\theta-x)}{\sin x}=\frac{\cos \gamma_{2}}{\cos \gamma_{1}} .
$$




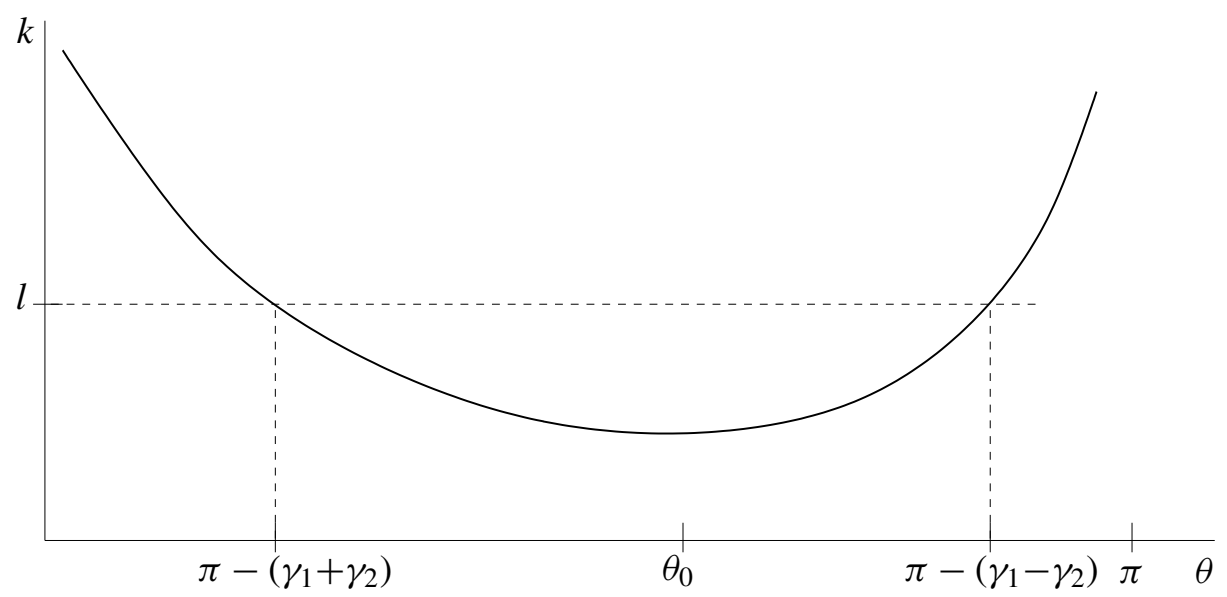

Figure 11. Lemma 3.3; $\alpha_{1}(\theta)<\pi / 2$ if $\theta<\theta_{0}, \alpha_{1}(\theta)>\pi / 2$ if $\theta>\theta_{0}$.

In particular, $\alpha_{1} \in[\alpha, \alpha+\pi / 2]$. If $\gamma_{2}=0$, then $\alpha_{2}:=0$.

Lemma 3.2. Let $\alpha_{1}(\theta)$ and $\alpha_{2}(\theta)$ be defined as in Lemma 3.1. Then $\alpha_{1}(\theta)$ increases strictly throughout the interval $0<\theta<\pi$. Letting $\theta_{0} \in(0, \pi)$ be the unique value such that $\cos \theta_{0}=-\cos \gamma_{2} / \cos \gamma_{1}$, there holds $\alpha_{1}\left(\theta_{0}\right)=\pi / 2 ; \alpha_{2}(\theta)$ increases strictly on $0<\theta<\theta_{0}$, and decreases strictly on $\theta_{0}<\theta<\pi$.

Lemma 3.3. (i) Let

$$
k(\theta):=\frac{\cos \gamma_{1}}{\sin \alpha_{1}(\theta)}\left(=\frac{\cos \gamma_{2}}{\sin \alpha_{2}(\theta)} \text { if } \gamma_{2} \neq \frac{\pi}{2}\right),
$$

where $\alpha_{1}$ and $\alpha_{2}$ are the partition of the opening angle $\theta$ as defined in Lemma 3.1. Then $k(\theta)$ decreases strictly in the interval $0<\theta<\theta_{0}$, and increases strictly in the interval $\theta_{0}<\theta<\pi$.

(ii) $k(\theta)=1$ if $\theta=\pi-\left(\gamma_{1}+\gamma_{2}\right) \operatorname{or} \theta=\pi-\left(\gamma_{2}-\gamma_{1}\right)$.

The proofs of these lemmata are elementary and we omit them here.

Remark. In the above lemmata we have to replace $\gamma_{1}$ by $\gamma_{2}$ and vice versa if the data are in (b); see Figure 9.

Lemma 3.3 implies that $0<k<1$ for data in (a), see Figure 9, if $\pi-\left(\gamma_{1}+\gamma_{2}\right)<$ $2 \alpha<\pi-\left(\gamma_{2}-\gamma_{1}\right)$ and $k>1$ if $2 \alpha<\pi-\left(\gamma_{1}+\gamma_{2}\right)$ or $2 \alpha>\pi-\left(\gamma_{2}-\gamma_{1}\right)$. That is, $k>1$ and $\alpha_{1}<\pi / 2$ if $\left(\gamma_{1}, \gamma_{2}\right)$ is from the intersection of (a) and $\mathcal{D}_{1}^{+}$.

We thus see that comparison hemispheres achieving the data $\left(\gamma_{1}, \gamma_{2}\right) \in \mathcal{D}_{1}^{+}$are disjoint from the vertex $O$ and no neighborhood $\mathcal{N}$ of $O$ can be covered by a single equatorial circle. Given a point of $\mathcal{N}$, we can follow the method used above 
for data in $\mathcal{R}$, however the radius of the comparison disk tends to zero as $O$ is approached, in accordance with $\xi=R k$, see Figure 10. We find:

Theorem 3.1. Instead of the uniform height estimate (2-2) for all solutions in $\mathcal{N}$, we obtain a growth estimate

$$
u(x, y)<M_{r / k}+O(1) \text { as } r \rightarrow 0,-\alpha_{2} \leq \varphi \leq \alpha_{2},
$$

where $r=\sqrt{x^{2}+y^{2}}$, for all solutions of (1-1) with data in $\mathcal{D}_{1}^{+}$, under Hypothesis $A^{+}$. Hypothesis $A^{-}$yields a corresponding inequality with reversed sign on the right and $<$ replaced by $>$.

In terms of order of magnitude, (3-1) cannot be improved, as one sees from the complete asymptotic development in a particular case, in Section $3 \mathrm{~A}$ of this paper. Still the information offered by (3-1) is incomplete in several respects. Notably, we may write $u^{+}=\max \{u(x, y), 0\}, u^{-}=\max \{-u(x, y), 0\}$ and obtain

Theorem 3.2. Under Hypothesis $A^{+}$and data in $\mathcal{D}_{1}^{+}$we have

$$
f^{+}(x, y, u(x, y)) \geq 2(k-1) / r,
$$

where $r=\sqrt{x^{2}+y^{2}}$. In particular, there is no solution $u(x, y)$ of $(1-1)$ in $\mathcal{N}$ for which

$$
f^{+}(x, y, u(x, y))=o(1 / r) .
$$

Proof. Suppose there is a graph solution $u$ defined in a neighborhood of the vertex $O$ for (1-1). Let the $x$-axis passing through $O$ which divides the angle $2 \alpha$ into $\alpha_{1}$ and $\alpha_{2}$ as given in Lemma 3.1, and let $\overline{A B}$ be a straight line perpendicular to the $x$-axis. As we have seen that $\alpha_{1}<\pi / 2$ so $\overline{A B}$ must meet $\Sigma_{1}$ and $\Sigma_{2}$, say at $A$ and $B$ respectively, let $\triangle=\triangle O A B, a=\overline{O A}, b=\overline{O B}$, and $\delta$ be the distance of $O$ to $\overline{A B}$, see Figure 12. Integrate (1-1) over $\triangle$ ( $\triangle$ will be contained in the domain of $u$ if $\delta$ is sufficiently small), using the definition of $k$, see Lemma 3.3 and the fact that $|T u| \leq 1$, we get

$$
\begin{aligned}
& \int_{\Delta} f(x, y, u(x, y)) d \Omega=\int_{\partial \Delta} v \cdot T u d s \\
= & a \cos \gamma_{1}+b \cos \gamma_{2}+\int_{\overline{A B}} v \cdot T u d s \\
= & k \overline{A B}+\int_{\overline{A B}} v \cdot T u d s \geq(k-1) \overline{A B}
\end{aligned}
$$

which yields

$$
f^{+}(x, y, u(x, y)) \delta \geq 2(k-1)
$$

for all $0<\delta<\delta_{0}$. Since $k-1$ is a positive constant, see Lemma 3.3, the theorem is proved. 


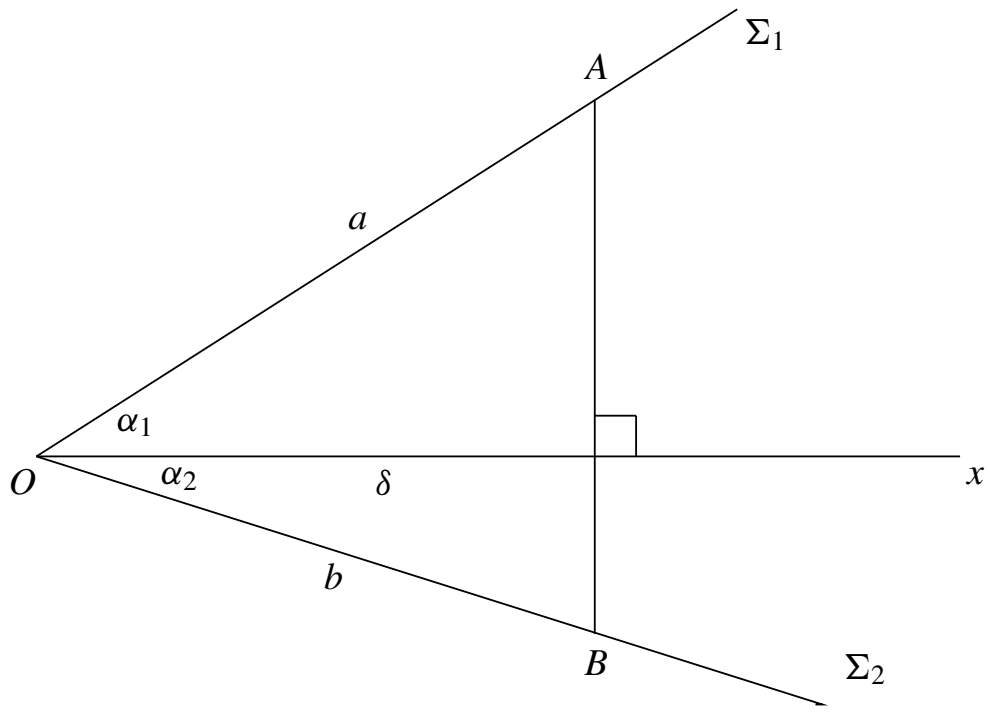

Figure 12. Proof of Theorem 3.2.

Remark. Note that no hypothesis was introduced as to behavior of $f^{-}(x, y, s)$, which could be arbitrary, even though no bound from below is assumed on the solution.

If $\mathrm{A}^{+}$is replaced by $\mathrm{A}^{-}$, with data again in $\mathcal{D}_{1}^{+}$, one obtains a much stronger growth bound. We find more generally:

Theorem 3.3. For data in $\mathcal{D}_{1}^{+}$, an upper hemisphere of radius $\delta>0$ can be constructed whose equatorial disk $D_{\delta}$ is as in Figure 13, with $D_{\delta} \cap \mathcal{N}=\Omega_{\delta} \subset \mathcal{W}$, and which cuts the boundary walls in angles minorizing the data. Under Hypothesis $A^{-}$ an estimate of the form (2-3) holds throughout $\Omega_{\delta}$. Under Hypothesis $B^{-}$every solution is bounded below in $\Omega_{\delta}$, depending only on $\delta$ and on its values on a compact interior subset of $\Omega_{\delta}$.

Thus in this case, solutions in the wedge domain $\mathcal{W}$ are bounded below, both for bounded and for unbounded $f$.

Proof. As we see from Figure 2, there is a wide choice of minorizing data from $\mathcal{R}$. For any such data one can, essentially, repeat the proof of (2-3). As a specific choice, we may construct a particular upper hemisphere cutting the wedge sides in angles $\gamma_{1}^{*}, \gamma_{2}^{*}$, and seek to choose these angles so that $\pi \geq \gamma_{1}^{*} \geq \gamma_{1}, \pi \geq \gamma_{2}^{*} \geq \gamma_{2}$. We may choose the configuration as in Figure 4 so that

$$
\gamma_{1}^{*}+\gamma_{2}^{*}=\pi-2 \alpha .
$$




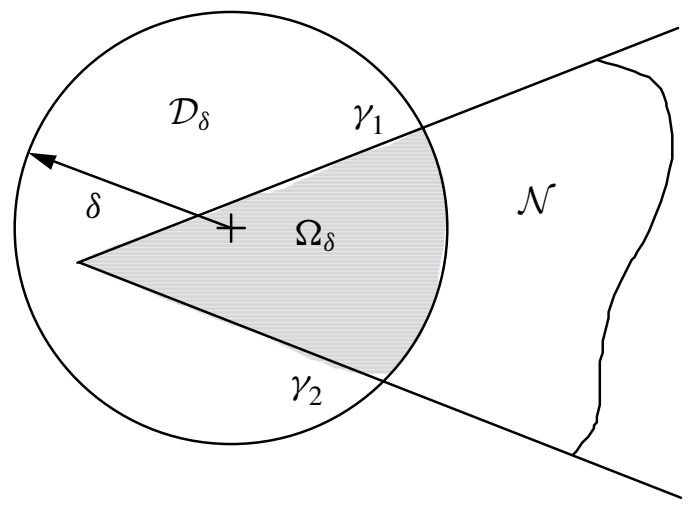

Figure 13. Neighborhood $\Omega_{\delta}$ for minorization; data in $\mathcal{D}_{1}^{+}$, Hypothesis $\mathrm{A}^{+}$.

We know that $\gamma_{1}+\gamma_{2}<\pi-2 \alpha$. If $\gamma_{1} \leq 2 \alpha$, choose $\gamma_{1}^{*}=2 \alpha+\epsilon$, where $0<\epsilon<$ $\min \left\{2 \alpha-\gamma_{1}, \pi-2 \alpha\right\}$. Then $\pi>\gamma_{1}^{*} \geq \gamma_{1}$, and $\gamma_{2}^{*}=2 \alpha-\gamma_{1}^{*}=\pi-\epsilon$. If $\gamma_{1}>2 \alpha$, set $\gamma_{1}^{*}=\gamma_{1}$. Then $\gamma_{2}^{*}=\pi+2 \alpha-\gamma_{1}>\pi+2 \alpha-\left(\pi-2 \alpha-\gamma_{2}\right)=4 \alpha+\gamma_{2}>\gamma_{2}$. Also $\gamma_{2}^{*} \equiv \pi+2 \alpha-\gamma_{1}^{*}<\pi$ since $\gamma_{1}^{*}>2 \alpha$. Thus the single sphere of radius $\delta$ serves as comparison surface from below, for all points of $\Omega_{\delta}$. The method used to prove (2-3) thus yields in this case that same estimate throughout $\Omega_{\delta}$.

The final statement of the theorem follows from the same reasoning used above and from $\left(\mathrm{a}^{\prime}\right)$ and $\left(\mathrm{b}^{\prime}\right)$ with data from $\mathcal{R}$.

For data $\left(\gamma_{1}, \gamma_{2}\right) \in \mathcal{D}_{1}^{-}$analogous statements are obtained by the same methods, however there is some permutation of signs. The relevant statements for each case are outlined in Table 1 of Section 5 below.

3A. Asymptotic expansion in the classical case. In the classical case the right hand side of (1-1) is $f(x, y, s)=\kappa s+\lambda$, where $\kappa$ and $\lambda$ are constants. In this section we assume that $\kappa$ is positive, that is, the gravitational field is directed downward. By adding a suitable constant to $u$, we may assume that $\lambda=0$, and equations in (1-1) become

$$
\begin{aligned}
\operatorname{div}(T u)(x, y) & =\kappa u(x, y) \quad \text { in } \Omega, \\
v \cdot T u & =\cos \gamma_{1} \quad \text { on } \Sigma_{1}, \\
v \cdot T u & =\cos \gamma_{2} \quad \text { on } \Sigma_{2} .
\end{aligned}
$$

The behavior of solutions of (3-2) for equal contact angles was first investigated by Concus and Finn $[1969,1974]$. In particular, they showed that $u \sim O(1 / r)$ as $r \rightarrow 0$, where $r$ is distance to the vertex. This result was later improved by Miersemann [1993] to arbitrary finite order of asymptotic expansion. We extend these results to differing contact angles for all data in the $\mathcal{D}_{1}$ domains. 
The following arguments follow the idea of Concus and Finn [1974]. From Theorems 3.1 and 3.3 it follows $c_{1} / r \leq u \leq c_{2} / r, 0<r<r_{0}$, with positive constants $c_{1}, c_{2}$.

To find a comparison function $v \sim O(1 / r)$ for equations (3-2) to characterize the behavior of $u$ near the corner, we observe first that the curvature of the function $1 / r$ goes to zero in an order of $O\left(r^{3}\right)$ as $r$ goes to zero and since the surface determined by $u$ is almost "flat" along the $z$-axis if $u=O(1 / r)$, we conclude that the mean curvature of $u$ is almost determined by the level curves. Noting that the mean curvature of $u$ is proportional to the height of $u$ by (3-2), we then have that the curvature of the level curves is almost constant. We recall that $\operatorname{div}(T u)(x, y)$ is twice the mean curvature of the surface given by $z=u(x, y)$.

On the other hand, since the surface determined by $u$ is almost flat along the $z$ axis, the contact angles between the surface and the planes vertical on $\Sigma_{1}$ and $\Sigma_{2}$ are almost determined by the level curves. Thus, it is natural to seek a comparison function $v$ which behaves like $O(1 / r)$ as $r$ goes to zero and whose level curves are circular arcs making contact angles $\gamma_{1}$ and $\gamma_{2}$ with $\Sigma_{1}$ and $\Sigma_{2}$ respectively.

Let the positive $x$-axis be the half line which cuts the wedge angle $2 \alpha$ into $\alpha_{1}$ and $\alpha_{2}$ as defined by Lemma 3.1 and let $C_{R}$ be the circle of Figure 10.

To find the comparison function $v$, let $C_{R(v)}$ be the circular $v$-level curve of $v$ and let $R(v)$ be the radius of $C_{R(v)}$, put

$$
\frac{1}{R(v)}=\kappa v
$$

The circle $C_{R(v)}$ meets $\Sigma_{1}$ and $\Sigma_{2}$ in angles $\gamma_{1}$ and $\gamma_{2}$, respectively, if $\xi=k R$ and $(\xi, 0)$ is the center of $C_{R(v)}$, see Lemma 3.3, and $C_{R(v)}$ is given by equation

$$
(x-\xi)^{2}+y^{2}=R(v)^{2} .
$$

Setting $x=r \cos \varphi, y=r \sin \varphi$ in polar coordinates, putting $\xi=k R$ in (3-4) and solve $R(v)$ from (3-4), we get

$$
\frac{1}{R(v)}=\frac{k \cos \varphi-\sqrt{1-k^{2} \sin ^{2} \varphi}}{r} .
$$

Then follows from (3-3) that

$$
v=\frac{k \cos \varphi-\sqrt{1-k^{2} \sin ^{2} \varphi}}{\kappa r}, \quad-\alpha_{2}<\varphi<\alpha_{1} .
$$

As introduced in [Miersemann 1993], let

$$
h_{-1}(\varphi)=\frac{1}{\kappa}\left(k \cos \varphi-\sqrt{1-k^{2} \sin ^{2} \varphi}\right)
$$


we obtain by the method in [Miersemann 1993] that all solutions of equations (3-2) have the same singular behavior and there is a complete asymptotic expansion independent of the particular solution considered. More precisely, we have

Theorem 3.4. For any nonnegative integer $m$ there exist positive constants $r_{0}, A$ and $m+1$ bounded analytic functions $h_{4 j-1}(\varphi),-\alpha_{2}<\varphi<\alpha_{1}, j=0, \ldots, m$, such that

$$
\left|u(r \cos \varphi, r \sin \varphi)-\sum_{j=0}^{m} h_{4 j-1}(\varphi) r^{4 j-1}\right| \leq A r^{4 m+3}
$$

in $\Omega_{r_{0}}$. Moreover, the constants $r_{0}$, A and the functions $h_{4 j-1}$ do not depend on the particular solution considered.

The function $h_{-1}$ is defined by (3-5) and the others are solutions of linear boundary value problems of second order.

Remark. A closer inspection of the proof of Theorem 3.4 shows that the asymptotic expansion is uniform with respect to $\left(\gamma_{1}, \gamma_{2}\right) \in \mathcal{D}_{1}^{+}$and $\gamma_{1}+\gamma_{1} \leq \pi-2 \alpha-\epsilon$, where $\epsilon>0$ is fixed.

\section{Reentrant corners}

In this case one sees immediately from the methods of Section 2A that under any of the hypotheses $\mathrm{A}^{+}, \mathrm{A}^{-}, \mathrm{B}^{+}, \mathrm{B}^{-}$all solutions are bounded at $O$; under the "A" hypotheses the bounds are explicitly known and are identical to those found in Section 2A. Nevertheless there can be great variety in the character of the solutions in particular subcases, as was shown in delicate investigations by Lancaster and Siegel [1997] and then by Shi [2006]. As is known (see, for exmaple [Korevaar 1980]) solutions corresponding to given data and corner angle can be continuous or discontinuous, depending on the global configuration. Following [Lancaster and Siegel 1997], we introduce the radial limits $R u$ corresponding to the angle $\vartheta$ between $\Sigma_{1}$ and a radial segment approaching $O$. These limits are shown in [Lancaster and Siegel 1997] to exist for every $\vartheta$ in the range $0<\vartheta<2 \alpha$. The authors of that paper restrict themselves to the discontinuous case; detailed analysis of the continuous eventuality awaits investigation. Figure 14 provides an analogue of Figure 2 which is appropriate for reentrant corners.

Following [Lancaster and Siegel 1997], we introduce the following descriptions of conceivable behavior of $R u$ at $O$.

C: $\quad$ continuous at $O$.

D: there exists an interval $0<\vartheta<\varepsilon_{1}$ in which $R u$ is constant, then an interval $\varepsilon_{1}<\vartheta<2 \alpha-\varepsilon_{2}$ in which $R u$ decreases monotonically, then $R u$ is again constant in $2 \alpha-\varepsilon_{2}<\vartheta<2 \alpha$. 


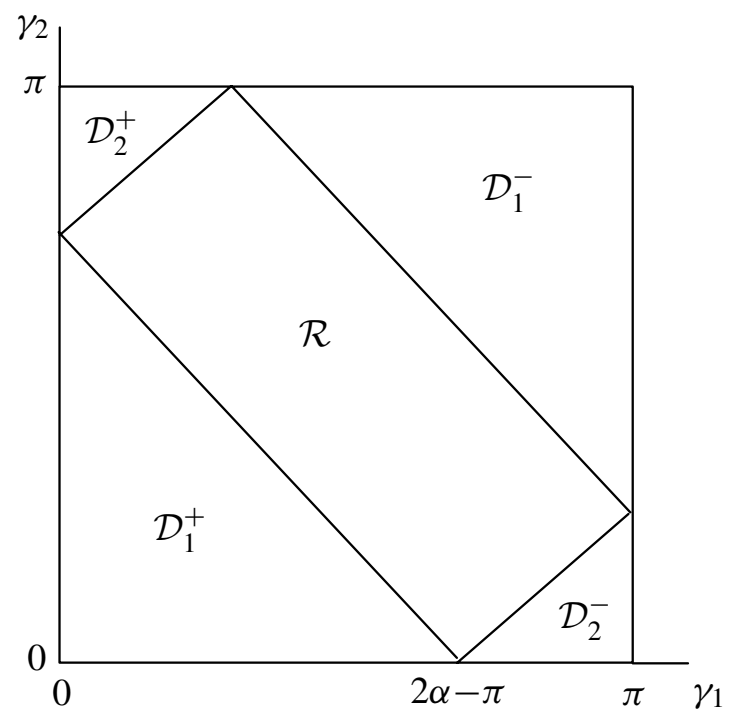

Figure 14. Data domains when $2 \alpha>\pi$.

I: $\quad$ same as D, with "decreases" replaced by "increases".

DI: $\quad R u$ is constant in $0<\vartheta<\varepsilon_{1}$, then decreases in $\varepsilon_{1}<\vartheta<\vartheta_{0}$, then is again constant in $\vartheta_{0}<\vartheta<\vartheta_{0}+\pi$, then increases in $\theta_{0}+\pi<\vartheta<2 \alpha-\epsilon_{2}$, and finally is once more constant in $2 \alpha-\varepsilon_{2}<\vartheta<2 \alpha$.

ID: same as DI, with "decreases" and "increases" interchanged.

Remarkably, these are the only types of behavior that can occur. Occurrence or nonoccurrence depends on the region of Figure 14 in which the data appear.

\section{Summary}

Joining the present material with that of [Lancaster and Siegel 1997, 1996], we are led to Tables 1 and 2 correlating the choices of data domain and growth hypothesis for $f$ with the consequent behavior of solutions. All types indicated actually do occur.

These tables indicate only in general terms what will happen, and do not encompass all cases. In some configurations more detailed information is contained implicitly in the supporting discussions above or in the cited references. When detailed or extensive information is needed, investigators may do well to consult that material. The first table does bring out some discontinuous transitions, such as that occurring when data change from $\mathcal{R}$ to $\mathcal{D}_{1}^{ \pm}$. The proof presented here for that behavior is, we think, an improvement over the initial one offered in [Concus and Finn 1996]. 

$\mathrm{A}^{+}$
$\mathrm{A}^{-}$
$\mathrm{A}^{+} \cup \mathrm{A}^{-} \cup \mathcal{M}$
$\mathrm{B}^{+}$
$\mathrm{B}^{-}$

$\mathcal{R} \quad u<M_{\delta}+\delta \quad u>-M_{\delta}-\delta$

C

Bdd above

Bdd below

No solution if

$\mathcal{D}_{1}^{+} \quad f^{+}=o(1 / r) . \quad u>-M_{\delta}-\delta$

$\exists$ solutions

for which

$f^{+}=O(1 / r)$

$\mathcal{L}_{1}^{+} \quad u<M_{\delta}+\delta \quad u>-M_{\delta}-\delta$

The neighboring

two columns on

No solution Bdd below the left both hold

$-M_{\delta}-\delta<u \quad$ No solution

Bdd below $<M_{\delta}+\delta$

No solution if The neighboring

$\mathcal{D}_{1}^{-} \quad u<M_{\delta}+\delta \quad f^{-}=o(1 / r) . \quad$ two columns on

$\exists$ solutions

for which

the left both

hold

$f^{-}=O(1 / r)$

$\mathcal{L}_{1}^{-} \quad u<M_{\delta}+\delta$

$u>-M_{\delta}-\delta$

$-M_{\delta}-\delta<u$ $<M_{\delta}+\delta$

$\mathcal{D}_{2}^{+} \quad u<M_{\delta}+\delta \quad u>-M_{\delta}-\delta$

D

Bdd above No solution

$\mathcal{L}_{2}^{+} \quad u<M_{\delta}+\delta \quad u>-M_{\delta}-\delta$

Not established

Bdd above

No solution

$\mathcal{D}_{2}^{-} \quad u<M_{\delta}+\delta$

$u>-M_{\delta}-\delta$

I

Bdd above

Bdd below

$\mathcal{L}_{2}^{-} \quad u<M_{\delta}+\delta \quad u>-M_{\delta}-\delta$

Not established

Bdd above

Bdd below

Bdd above

Bdd below

Bdd above

Bdd below

Table 1. Protruding corner $0<2 \alpha<\pi$.
$\mathrm{A}^{+}$
$\mathrm{A}^{-}$
$\mathrm{A}^{+} \cup \mathrm{A}^{-} \cup \mathcal{M}$
$\mathrm{B}^{+}$
$\mathrm{B}^{-}$

$\mathcal{R} \quad u<M_{\delta}+\delta \quad u>-M_{\delta}-\delta$

$C, D, I$

Bdd above

Bdd below

$\mathcal{D}_{1}^{+} \quad u<M_{\delta}+\delta \quad u>-M_{\delta}-\delta$

$D I, D, I$

Bdd above

Bdd below

$\mathcal{L}_{1}^{+} \quad u<M_{\delta}+\delta \quad u>-M_{\delta}-\delta$

Not established

Bdd above

Bdd below

$\mathcal{D}_{1}^{-} \quad u<M_{\delta}+\delta$

$u>-M_{\delta}-\delta$

$I D, I, D$

Bdd above

Bdd below

$\mathcal{L}_{1}^{-} \quad u<M_{\delta}+\delta \quad u>-M_{\delta}-\delta$

Not established

Bdd above

Bdd below

$\mathcal{D}_{2}^{+} \quad u<M_{\delta}+\delta \quad u>-M_{\delta}-\delta$

D

Bdd above

Bdd below

$\mathcal{L}_{2}^{+} \quad u<M_{\delta}+\delta \quad u>-M_{\delta}-\delta$

Not established

Bdd above

Bdd below

$\mathcal{D}_{2}^{-} \quad u<M_{\delta}+\delta \quad u>-M_{\delta}-\delta$

I

Bdd above

Bdd below

$\mathcal{L}_{2}^{-} \quad u<M_{\delta}+\delta$

$u>-M_{\delta}-\delta$

Not established

Bdd above

Bdd below

Table 2. Reentrant corner $\pi<2 \alpha<2 \pi$. 


\section{Acknowledgment}

The second author is indebted to the Max-Planck-Institut für Mathematik in den Naturwissenschaften, Leipzig, for its hospitality during preparation of the manuscript.

\section{References}

[Athanassenas and Finn 2006] M. Athanassenas and R. Finn, "Compressible fluids in a capillary tube”, Pacific J. Math. 224:2 (2006), 201-229. MR 2007f:76031 Zbl 1126.76049

[Concus and Finn 1969] P. Concus and R. Finn, "On the behavior of a capillary surface in a wedge", Proc. Natl. Acad. Sci. USA 63 (1969), 292-299. Zbl 0219.76104

[Concus and Finn 1974] P. Concus and R. Finn, "On capillary free surfaces in a gravitational field", Acta Math. 132 (1974), 207-223. MR 58 \#32327c Zbl 0382.76005

[Concus and Finn 1996] P. Concus and R. Finn, "Capillary wedges revisited", SIAM J. Math. Anal. 27:1 (1996), 56-69. MR 96m:76006 Zbl 0843.76012

[Emmer 1973] M. Emmer, "Esistenza, unicità e regolarità nelle superfici de equilibrio nei capillari”, Ann. Univ. Ferrara Sez. VII (N.S.) 18 (1973), 79-94. MR 49 \#1281 Zbl 0275.49005

[Finn 1986] R. Finn, Equilibrium capillary surfaces, Grundlehren der Mathematischen Wissenschaften 284, Springer, New York, 1986. MR 88f:49001 Zbl 0583.35002

[Finn 1996] R. Finn, "Local and global existence criteria for capillary surfaces in wedges", Calc. Var. Partial Differential Equations 4:4 (1996), 305-322. MR 97f:53005 Zbl 0872.76017

[Gauss 1830] C. F. Gauss, "Principia Generalia Theoriae Figurae Fluidorum", Comment. Soc. Regiae Scient. Gottingensis Rec. 7 (1830).

[Huff and McCuan 2006] R. Huff and J. McCuan, "Scherk-type capillary graphs", J. Math. Fluid Mech. 8:1 (2006), 99-119. MR 2006k:76022 Zbl 1125.76012

[Korevaar 1980] N. J. Korevaar, "On the behavior of a capillary surface at a re-entrant corner", Pacific J. Math. 88:2 (1980), 379-385. MR 82c:49033 Zbl 0475.49034

[Lancaster $\geq 2008]$ K. Lancaster, "A proof of the Concus-Finn conjecture”, Submitted.

[Lancaster and Siegel 1996] K. E. Lancaster and D. Siegel, "Behavior of a bounded non-parametric $H$-surface near a reentrant corner”, Z. Anal. Anwendungen 15:4 (1996), 819-850. MR 97m:53011 Zbl 0866.35046

[Lancaster and Siegel 1997] K. E. Lancaster and D. Siegel, "Correction to: "Existence and behavior of the radial limits of a bounded capillary surface at a corner"”, Pacific J. Math. 179:2 (1997), 397-402. MR 98g:58030b Zbl 0866.76018

[Laplace 1805, 1806] P. S. Laplace, Traité de mécanique céleste, suppléments au Livre X, vol. 4, Gauthier-Villars, Paris, 1805, 1806.

[Lieberman 1988] G. M. Lieberman, "Hölder continuity of the gradient at a corner for the capillary problem and related results”, Pacific J. Math. 133 (1988), 115-135. MR 89h:35050 Zbl 0669.35034

[Miersemann 1989] E. Miersemann, "On the behaviour of capillaries at a corner", Pacific J. Math. 140:1 (1989), 149-153. MR 90j:35081 Zbl 0683.49013

[Miersemann 1993] E. Miersemann, "Asymptotic expansion at a corner for the capillary problem: the singular case", Pacific J. Math. 157:1 (1993), 95-107. MR 93m:35039 Zbl 0796.76020

[Miersemann 2008] E. Miersemann, "Kapillarflächen”, Ber. Verh. Sächs. Akad. Wiss. Leipzig. Math.Natur. Kl. 4 (2008). 
[Shi 2006] D. Shi, “Capillary surfaces at a reentrant corner", Pacific J. Math. 224:2 (2006), 321-353. MR 2008a:58013 Zbl 1125.76013

[Simon 1980] L. Simon, "Regularity of capillary surfaces over domains with corners", Pacific J. Math. 88:2 (1980), 363-377. MR 82d:49043 Zbl 0467.35022

[Tam 1986] L.-F. Tam, "On existence criteria for capillary free surfaces without gravity", Pacific J. Math. 125:2 (1986), 469-485. MR 88a:49023 Zbl 0604.49030

[Taylor 1712] B. Taylor, "Concerning the ascent of water between two glass planes", Philos. Trans. Roy. Soc. London 27 (1712), 538.

[Young 1805] T. Young, "An essay on the cohesion of fluids", Philos. Trans. Roy. Soc. London 95 (1805), 65-87.

Received January 12, 2008.

\section{Jin TZU CHEN}

DEPARTMENT OF MATHEMATICS

NATIONAL TAIWAN UNIVERSITY

TAIPEI, TAIWAN

REPUBLIC OF CHINA

jintzuchen@ntu.edu.tw

\section{ROBERT FINN}

MATHEMATICS DEPARTMENT

STANFORD UNIVERSITY

STANFORD, CA 94305-2125

UNITED STATES

finn@math.stanford.edu

\section{ERICH MiERSEMANN}

Mathematisches Institut

UNIVERSITÄT LEIPZIG

D-04109 LEIPZIG

GERMANY

miersemann@math.uni-leipzig.de 